\title{
Time-Frequency Analysis and Target Recognition of HRRP Based on CN-LSGAN, STFT, and CNN
}

\author{
Jianghua Nie $\mathbb{D}^{1},{ }^{1}$ Yongsheng Xiao $\mathbb{D D}^{1,2}$ Lizhen Huang $\mathbb{D}^{1},{ }^{1}$ and Feng $\mathrm{Lv}^{3}$ \\ ${ }^{1}$ Key Laboratory of Jiangxi Province for Image Processing and Pattern Recognition, School of Information Engineering, \\ Nanchang Hangkong University, Nanchang 330063, China \\ ${ }^{2}$ National Engineering Laboratory for Video Technology, School of Electronics Engineering and Computer Science, \\ Peking University, Beijing 100871, China \\ ${ }^{3}$ Henan Aerospace Hydraulic Pneumatic Technology Company Limited, \\ China Aerospace Science and Industry Corporation Limited, Zhengzhou 451191, China
}

Correspondence should be addressed to Yongsheng Xiao; xysfly@nuaa.edu.cn

Received 18 December 2020; Revised 15 March 2021; Accepted 26 March 2021; Published 13 April 2021

Academic Editor: Chenquan Gan

Copyright (C) 2021 Jianghua Nie et al. This is an open access article distributed under the Creative Commons Attribution License, which permits unrestricted use, distribution, and reproduction in any medium, provided the original work is properly cited.

\begin{abstract}
Aiming at the problem of radar target recognition of High-Resolution Range Profile (HRRP) under low signal-to-noise ratio conditions, a recognition method based on the Constrained Naive Least-Squares Generative Adversarial Network (CN-LSGAN), Short-time Fourier Transform (STFT), and Convolutional Neural Network (CNN) is proposed. Combining the Least-Squares Generative Adversarial Network (LSGAN) with the Wasserstein Generative Adversarial Network with Gradient Penalty (WGAN-GP), the CN-LSGAN is presented and applied to the HRRP denoise. The frequency domain and phase features of HRRP are gained by STFT in order to facilitate feature learning and also match the input data format of the CNN. These experimental results show that the CN-LSGAN has better data augmentation performance and can effectively avoid the model collapse compared to the generative adversarial network (GAN) and LSGAN. Also, the method has better recognition performance than the one-dimensional CNN method and the Long Short-Term Memory (LSTM) network method.
\end{abstract}

\section{Introduction}

HRRP is the vector sum of all scatters electromagnetic echoes, which can reflect the geometric structure, scatters point distribution, and other characteristics of the target. Also, it is easy to be obtained and processed, so the radar target recognition of HRRP has become a research hotspot that has attracted much attention in the academic community [1-11]. In recent years, with the large-scale rise of deep neural networks, deep learning provides new ideas for the research of radar automatic target recognition (RATR) of HRRP. Compared to the traditional recognition method, the HRRP target recognition method of deep learning can avoid the excessive use of manual rules to extract the target features and acquire high-order features. The recognition rate of the Deep Belief Network (DBN) reached $92.8 \%$ [12]; the CNN was also applied to RATR [13, 14], and the recognition rate was greatly improved; the Recurrent Neural
Network (RNN) [15] and LSTM [16] had both achieved good recognition rates. However, in these actual application scenarios, HRRP acquired contain noise, which affects the amplitude of HRRP. When the signal-to-noise ratio of the data is low, the amplitude of these data differs greatly from the amplitude of the real data, and when the features are extracted, the extracted features also differ greatly from the features of the real data, thus interfering with the recognition results. So, HRRP needs to be enhanced in order to improve the signal-to-noise ratio. The GAN is a deep-learning model widely applied to data augmentation, and it was used for image blind denoising [17] and speech augmentation $[18,19]$. But, it has not been applied to HRRP augmentation. HRRP is denoised only by an Auto Encoder (AE) [20].

To solve the problem of radar target recognition of HRRP under low signal-to-noise ratio circumstances, we proposed a new kind of generative model, called the 
CN-LSGAN. The CN-LSGAN can generate data similar to the real data with low signal-to-noise data as input, thus improving the signal-to-noise ratio of the data. In addition, in order to simulate the noisy HRRP data in actual application scenarios, we added different degrees of Gaussian white noise to the real data and then used the CN-LSGAN to enhance the data to improve the SNR. Then, we used the obtained data for target recognition. In order to improve the recognition effect as much as possible, time-frequency analysis is performed on the data before the recognition, and the data are converted from one-dimensional data to twodimensional data. The obtained time-frequency data contain more features. In the target recognition task, the more the data features, the better the recognition effect is generally. Finally, the obtained time-frequency data are recognized by the $\mathrm{CNN}$, and the recognition result is obtained. In this paper, the following contributions have been achieved:

(1) Improve the LSGAN to solve the model collapse problem

(2) Improve the signal-to-noise ratio of HRRP data by the CN-LSGAN

(3) Use the combination of STFT time-frequency analysis and shallow network CNN architecture to test the recognition rate of the enhanced data by CN-LSGAN

The rest of this paper is organized as follows: The second part introduces the proposed method in detail, mainly including the illustration of the CN-LSGAN, STFT, and CNN; the third part chiefly focuses on the experimental results and analysis; and the fourth part concludes the paper.

\section{Time-Frequency Analysis and Target Recognition of HRRP Based on the CN-LSGAN, STFT, and CNN}

2.1. Design of the CN-LSGAN. The GAN is a generative model designed by Goodfellow [21]. The model consists of a generator $(G)$ and a discriminator $(D)$, as shown in Figure 1.

The input of $D$ is real data or $G(z)$, and the output of $D$ is 1 or 0 . The adversarial training is carried out between $D$ and $G$, so that $G(z)$ approaches the real data. When $D$ cannot determine the source of the input after continuous adversarial training and iterative optimization, it is considered that $G$ has learned the real data distribution.

The loss function of the GAN is

$$
\begin{aligned}
\min _{G} \max _{D} V(D, G)= & E_{x \sim P_{\text {data }}(x)}[\log D(x)] \\
& +E_{z \sim P_{z}(z)}[\log 1-D(G(z))],
\end{aligned}
$$

where $E$ is the expectation of the distribution function, $P_{\text {data }}$ is the real data distribution, and $P_{z}$ is the noise distribution.

The real data are the clean HRRP, and the input of $G$ is the noisy HRRP, as is shown in Figure 2. Through the adversarial training, the data generated by $G$ are similar to the clean HRRP, and then, the denoise purpose is achieved.

The LSGAN [22] is used instead of the GAN in this paper, and the loss function of the LSGAN is shown as follows:

$$
\left\{\begin{array}{l}
\min _{D} V(D, G)=E_{x \sim P_{\text {data }}(x)}\left[(D(x)-1)^{2}\right]+E_{z \sim P_{z}(z)}\left[(D(G(z)))^{2}\right] \\
\min _{G} V(D, G)=E_{z \sim P_{z}(z)}\left[(D(G(z))-1)^{2}\right]
\end{array}\right.
$$

Compared to the GAN, the LSGAN uses least-squares loss in place of cross-entropy loss, which effectively solves the gradient disappearance problem during the model training stage. However, the LSGAN also has shortcomings; the LSGAN has a large output freedom degree and is prone to model collapse. These problems will lead to poor quality and low diversity of generated samples. In terms of these problems, a constraint item is, respectively, added to the loss function of the LSGAN's $D$ and $G$, and the two constraint items are shown as follows:

$$
\begin{aligned}
& \xi(D)=E_{\widehat{x} \sim p_{\widehat{x}}}\left[\left(\left\|\nabla_{\widehat{x}} D(\widehat{x})\right\|_{2}-1\right)^{2}\right], \\
& \xi(G)=E_{z-p_{z}(z)}\left[(G(z)-x)^{2}\right] .
\end{aligned}
$$

Through the improvement of the LSGAN, formula (2) can be rewritten as follows:

$$
\left\{\begin{array}{l}
\min _{D} V(D, G)=E_{x \sim P_{\text {data }}(x)}\left[(D(x)-1)^{2}\right]+E_{z \sim P_{z}(z)}\left[(D(G(z)))^{2}\right]+\lambda_{1} E_{\widehat{x} \sim P_{\widehat{x}}}\left[\left(\left\|\nabla_{\widehat{x}} D(\widehat{x})\right\|_{2}-1\right)^{2}\right] \\
\min _{G} V(D, G)=E_{z \sim P_{z}(z)}\left[(D(G(z))-1)^{2}\right]+\lambda_{2} E_{z \sim P_{z}(z), x \sim P_{\text {data }}(x)}\left[(G(z)-x)^{2}\right] .
\end{array}\right.
$$




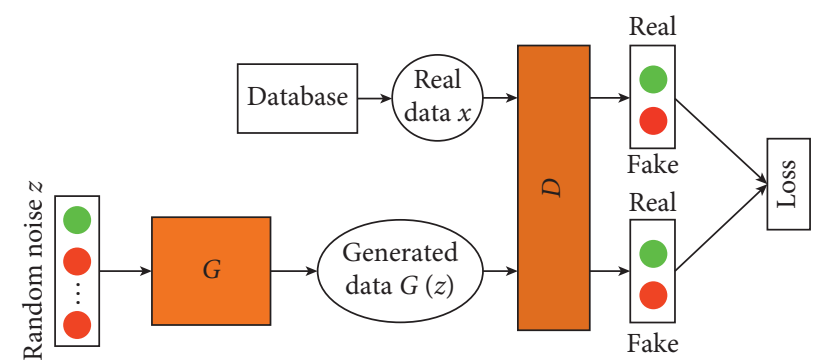

FIgURE 1: Schematic diagram of the GAN.

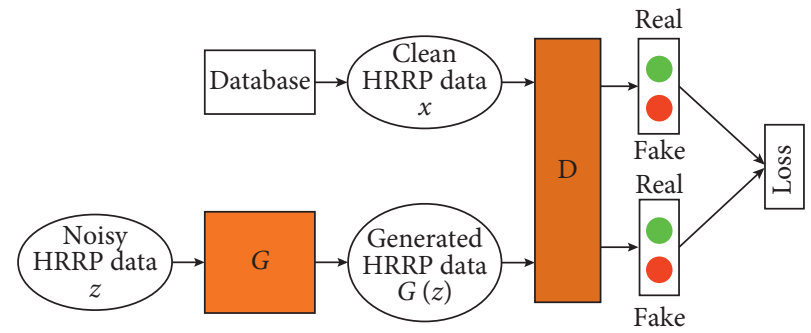

Figure 2: Schematic diagram of the GAN used for HRRP denoise.

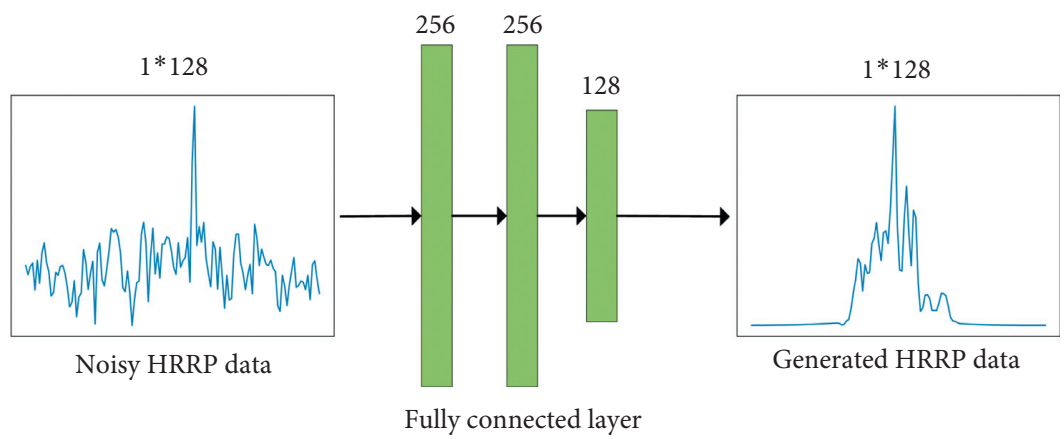

Figure 3: Schematic diagram of G.

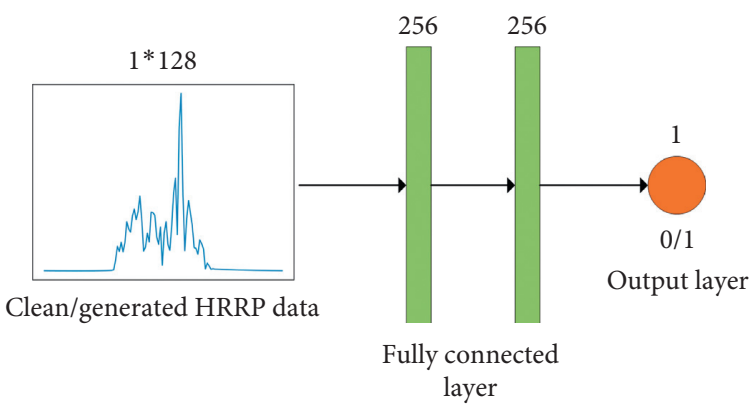

FIgURE 4: Schematic diagram of D.

In formula (5), $\lambda_{1}$ and $\lambda_{2}$ represent the adjustment weights, which adjust $\xi(D)$ and $\xi(G)$, respectively. $\xi(D)$ can solve the model collapse, and $\xi(G)$ can reduce the output freedom degree of G. The LSGAN with the abovementioned constraint items is called the CN-LSGAN.

Both $G$ and $D$ of the CN-LSGAN adopt the fully connected network structure, as shown in Figures 3 and 4 .
2.2. Time-Frequency Analysis Based on STFT. Time-domain analysis of HRRP only characterizes the amplitude of the signal, which only provides limited information for feature learning. In contrast, the spectral feature is a two-dimensional (time and frequency) representation of HRRP, which includes the frequency-domain characteristics of the target and reflects the phase information. STFT is used for the 


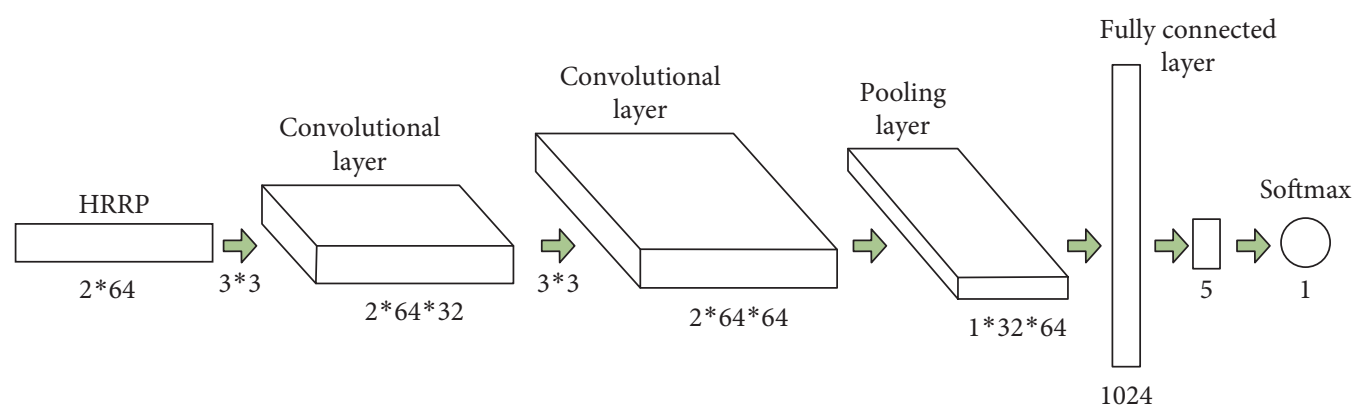

FIgURE 5: Schematic diagram of the CNN.
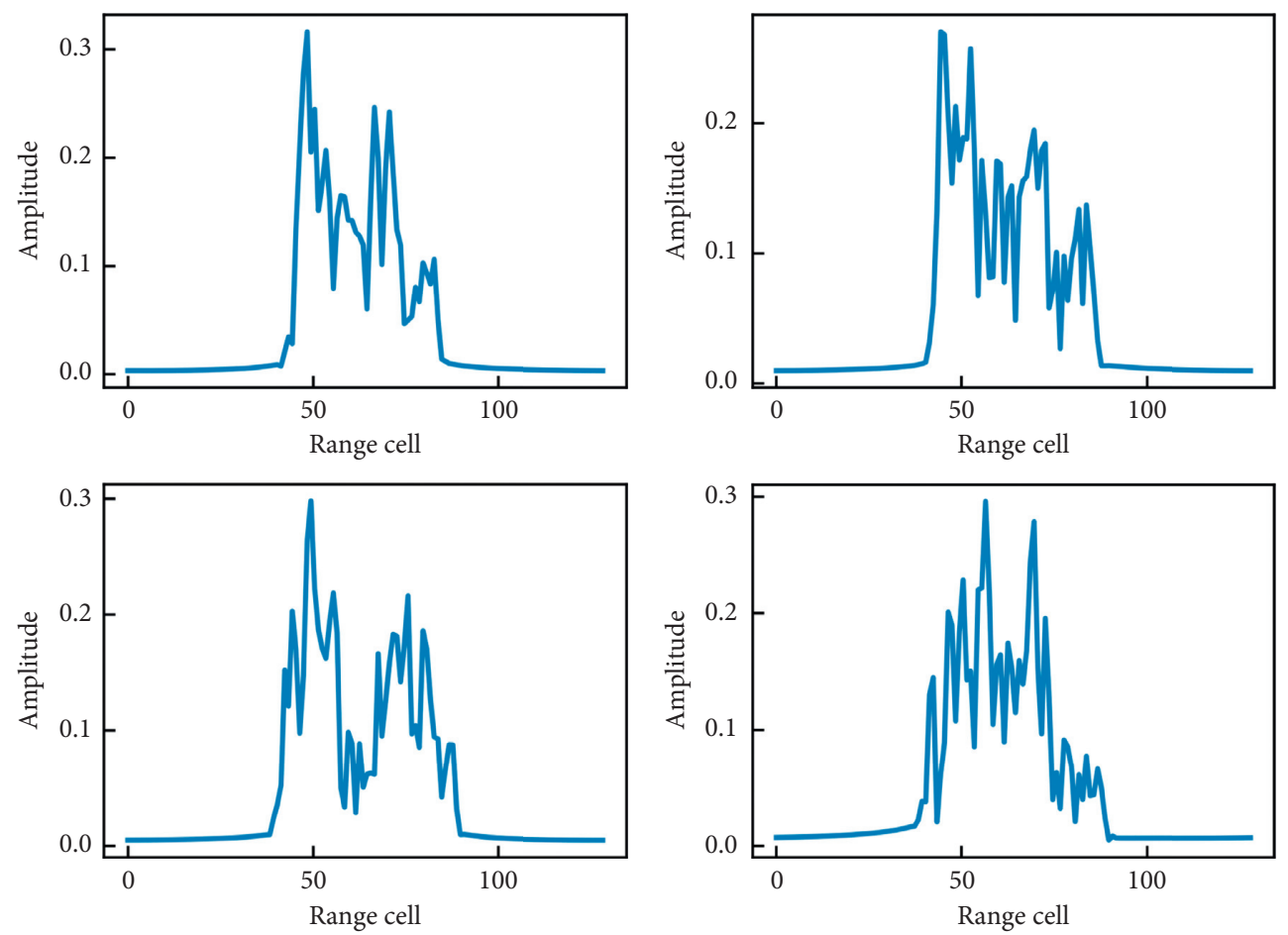

Figure 6: Four different data samples in dataset A.

time-frequency analysis of HRRP, and the window function is Hanning window. The width of the window affects the relative resolution of the time domain and frequency domain. In order to make a trade-off between the time-domain resolution and the frequency-domain resolution, the adjacent signal segments are superimposed on each other. According to the HRRP characteristics, the overlapping length between adjacent signal segments is selected to be half of the length of each segment, and Hanning windows with window lengths of 32, 16, 8, and 4 are used for STFT.

2.3. Design of the $C N N$. The dimension of the time-frequency data obtained by the time-frequency analysis for HRRP is (2, 64 ), and the $\mathrm{CNN}$ is designed according to the dimension. The model consists of two convolutional layers, a pooling layer, two fully connected layers, and a SoftMax classifier, and the sizes of the convolutional kernel of two convolutional layers are $3 * 3$, as shown in Figure 5 .

\section{Experimental Results and Analysis}

3.1. Experimental Data. The clean HRRP are gained by the electromagnetic simulation technology, which has simulated five kinds of aircraft (Su27, F16, M2000, J8II, and J6). There are 18,000 samples for each aircraft, so there are a total of 90,000 samples. In order to verify the effectiveness of the denoise method based on the CN-LSGAN, Gaussian white noise is added to the clean HRRP, and six kinds of noisy HRRP data with signal-to-noise ratios of $0 \mathrm{~dB}, 5 \mathrm{~dB}, 10 \mathrm{~dB}, 15 \mathrm{~dB}, 20 \mathrm{~dB}$, and $25 \mathrm{~dB}$ are obtained, where, the clean HRRP is called dataset A, the noisy HRRP is called dataset B, and the HRRP generated by the generative model is called dataset $C$; these three datasets are divided into a training set and test set at a ratio of 9:1.

3.2. HRRP Denoise. Figure 6 shows four samples of dataset $\mathrm{A}$, and Figure 7 shows four samples of dataset B with a signal-to-noise ratio of $10 \mathrm{~dB}$. 

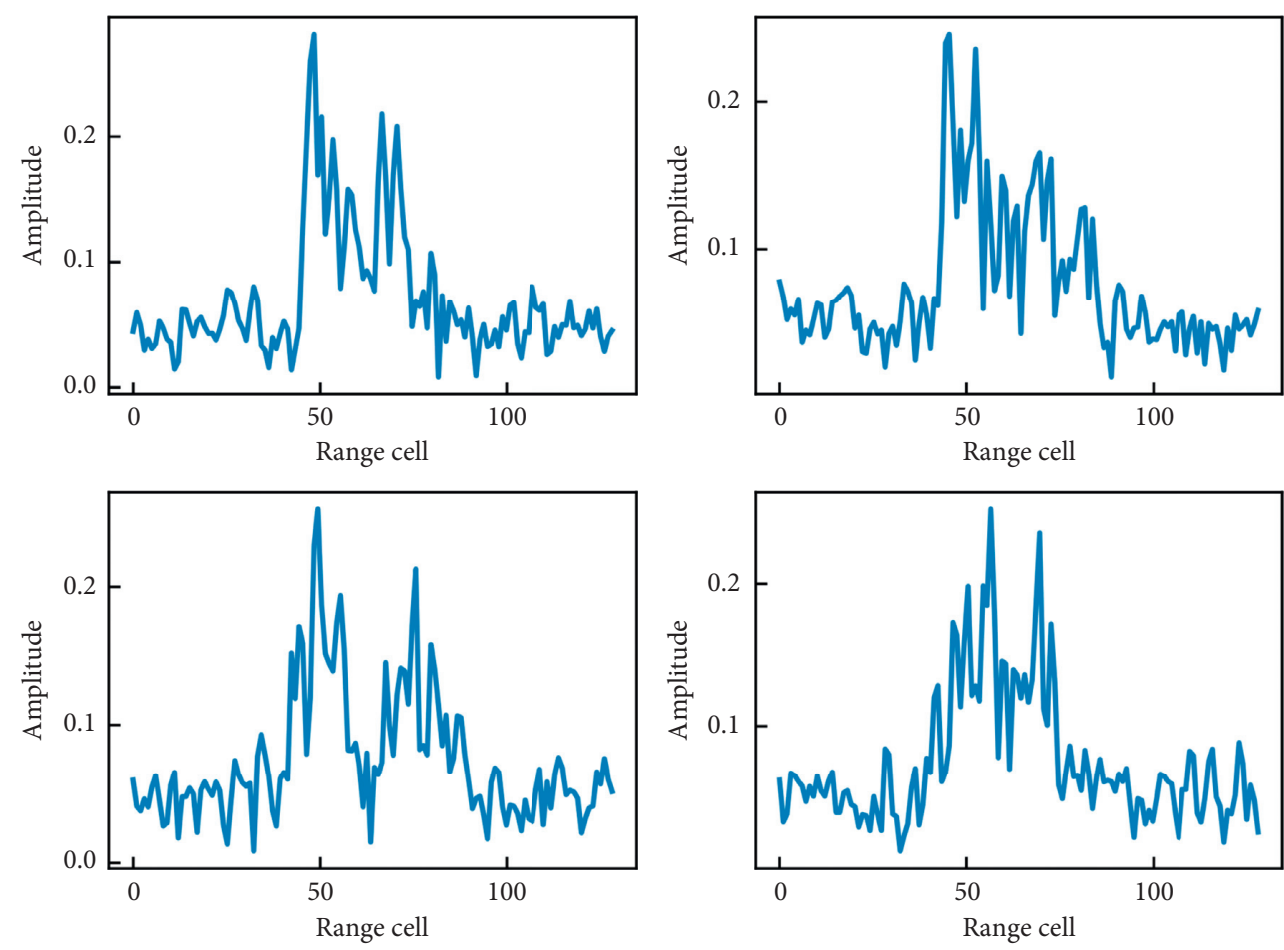

FigURE 7: Four different data samples in dataset B with a signal-to-noise ratio of 10db.
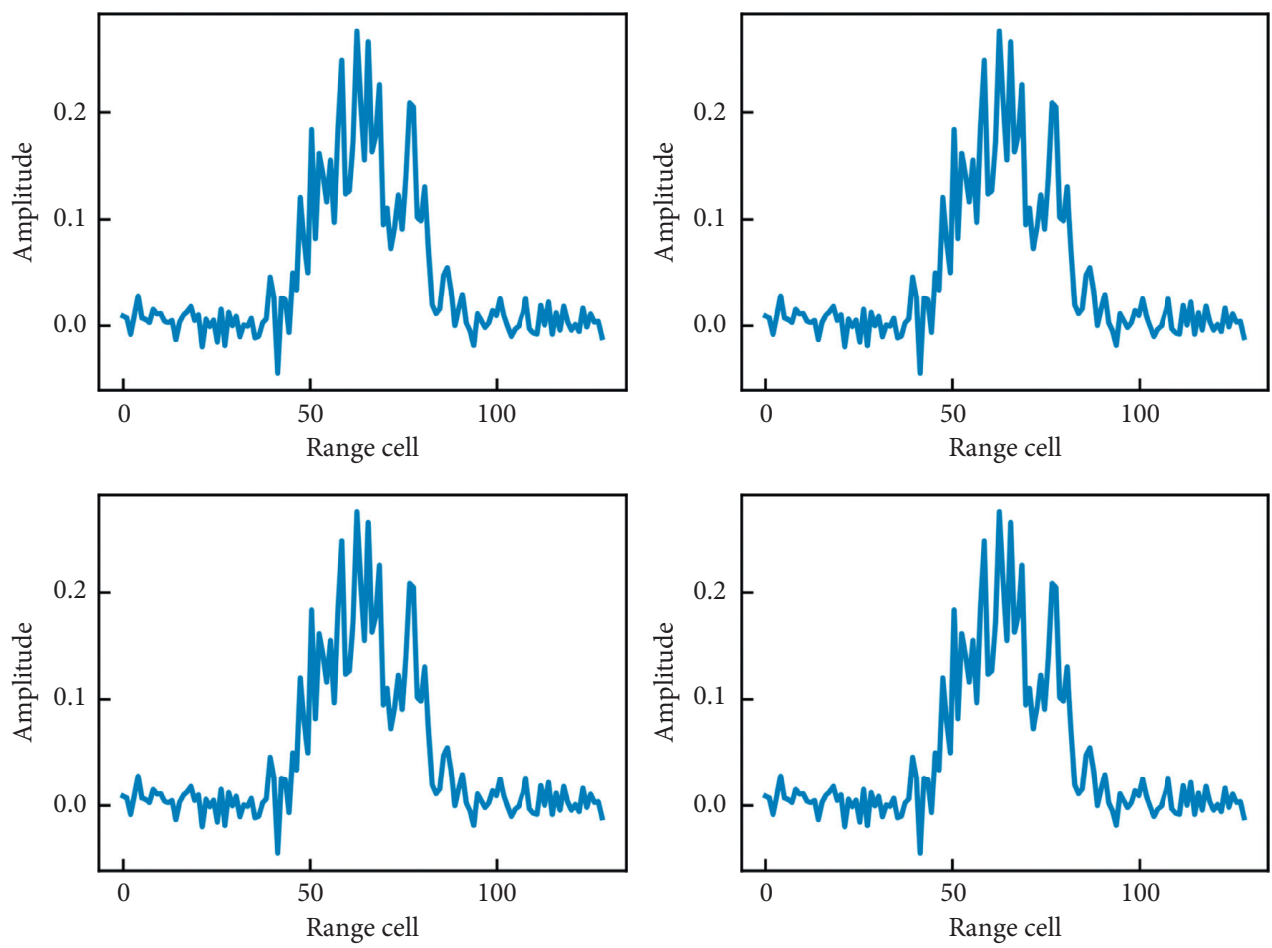

FIGURE 8: Four data samples in dataset (C), and dataset $\mathrm{C}$ is generated by the GAN.

Dataset $B$ with a signal-to-noise ratio of $10 \mathrm{~dB}$ is input to the GAN, LSGAN, and CN-LSGAN, respectively. Three kinds of data are generated, and they are shown in Figures 8-10.
Cosine similarity and Mean Square Error (MSE) among these data samples are technical indexes which indicate the probability of model collapse. They are calculated, respectively, for three kinds of data generated by the GAN, 

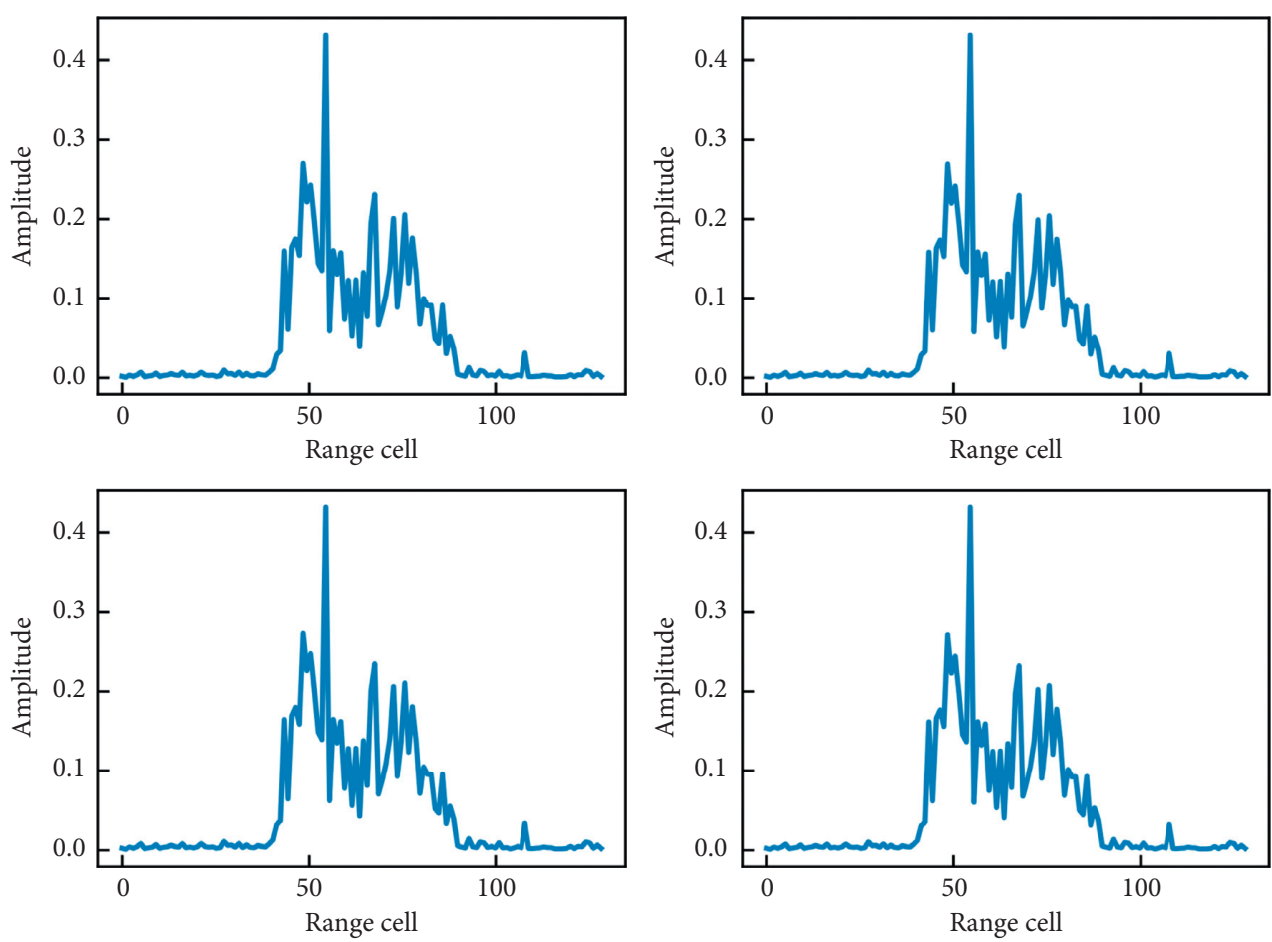

FIgUre 9: Four data samples in dataset (C), and dataset $C$ is generated by the LSGAN.
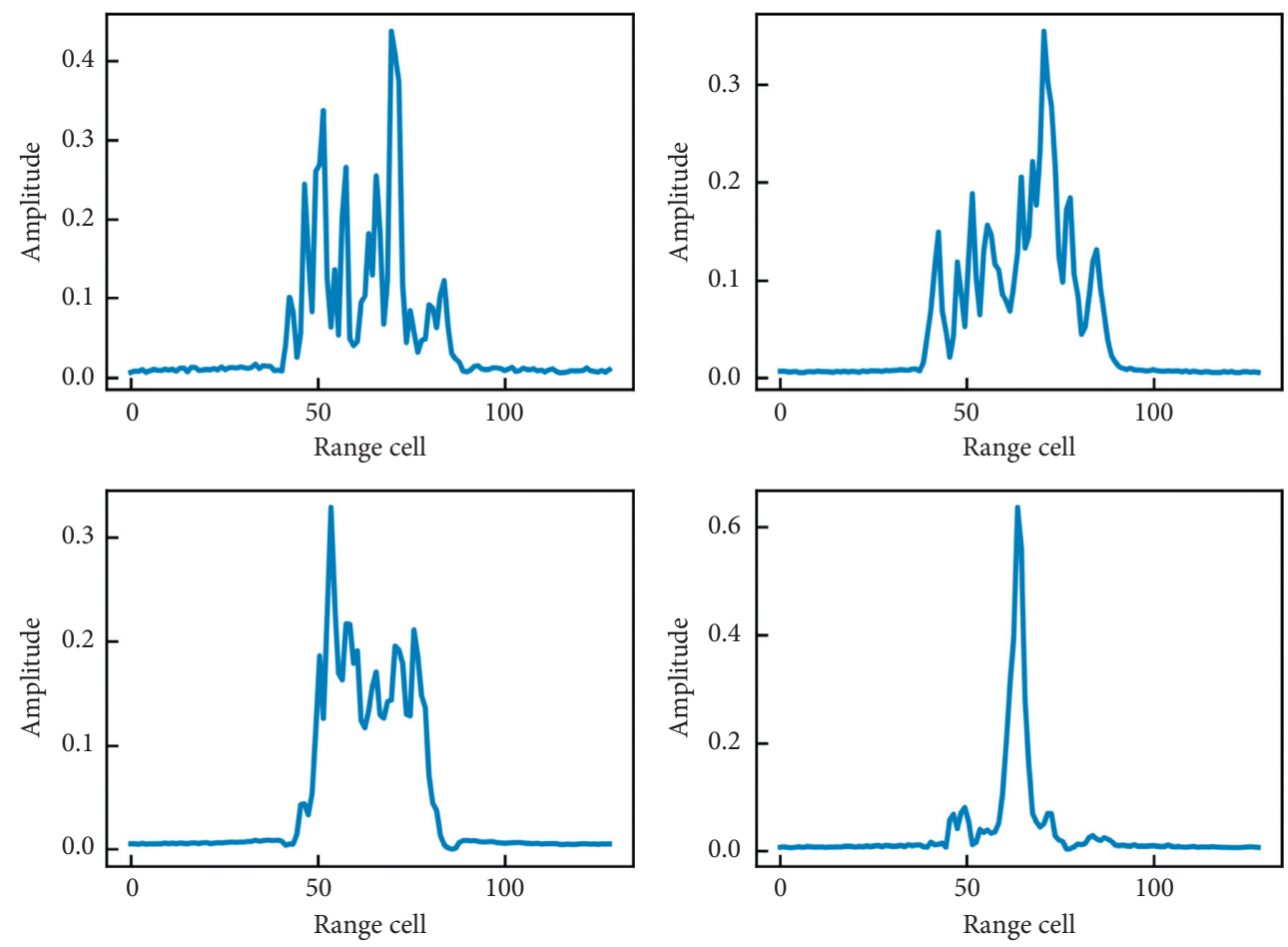

Figure 10: Four data samples in dataset (C), and dataset $\mathrm{C}$ is generated by the CN-LSGAN.

LSGAN, and CN-LSGAN. The calculated data are shown in Table 1.

When cosine similarity is equal to 1 or the MSE is equal to 0 , it means that the data samples in a certain dataset are very similar, which means that model collapse has occurred. From Table 1, it can be seen that the cosine similarity of the data generated by the GAN and LSGAN is both 1.0 and the MSE is equal to or very close to 0 , which means that the data 
TABLE 1: Sample correlation.

\begin{tabular}{lcc}
\hline Model & Cosine similarity & MSE \\
\hline GAN & 1.0 & 0 \\
LSGAN & 1.0 & $1.7984 \mathrm{e}-6$ \\
CN-LSGAN & 0.8457 & $1.9800 \mathrm{e}-3$ \\
\hline
\end{tabular}

TABLE 2: Quality evaluation.

\begin{tabular}{lcr}
\hline Model & GRA & PCC \\
\hline Noisy HRRP data & 0.8056 & 0.6935 \\
GAN & 0.8491 & 0.7749 \\
LSGAN & 0.8668 & 0.8086 \\
CN-LSGAN & 0.9443 & 0.8061 \\
\hline
\end{tabular}

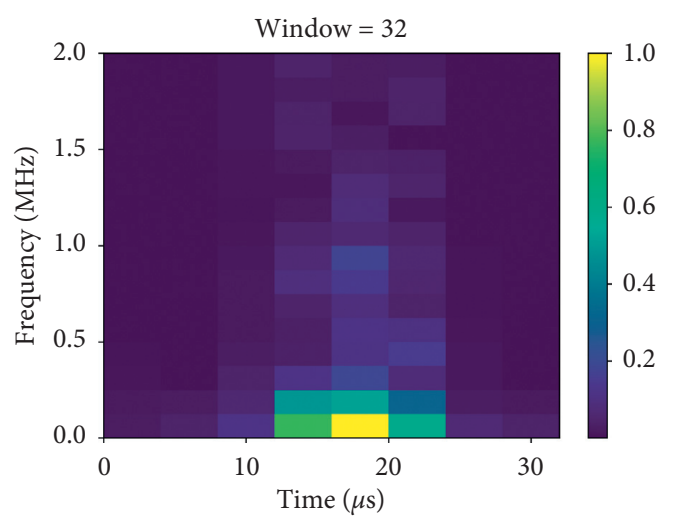

(a)

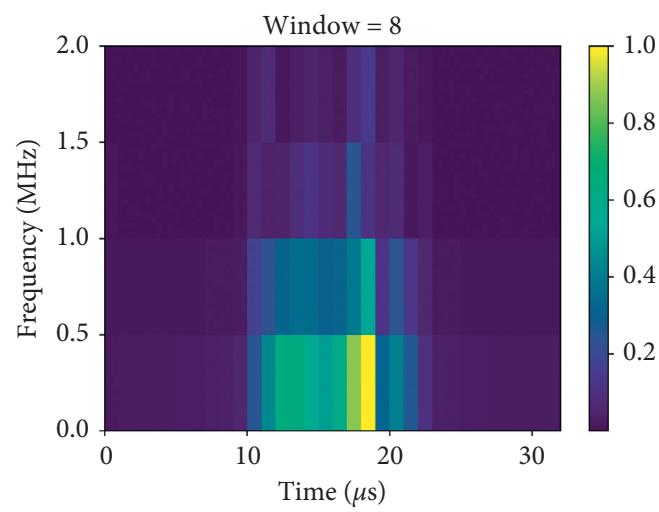

(c)

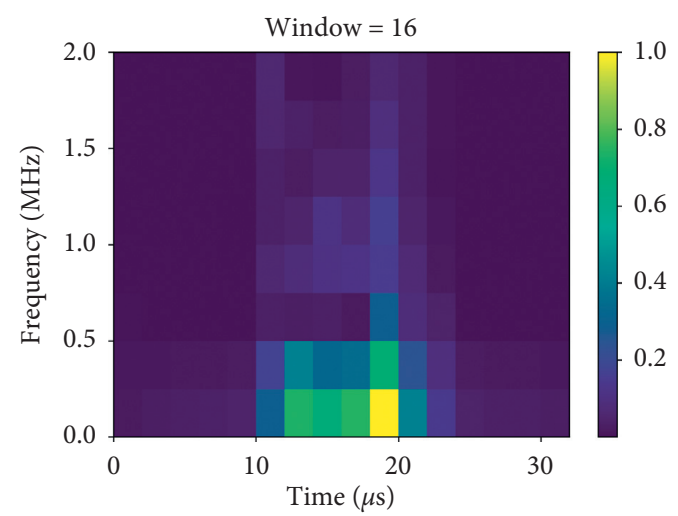

(b)

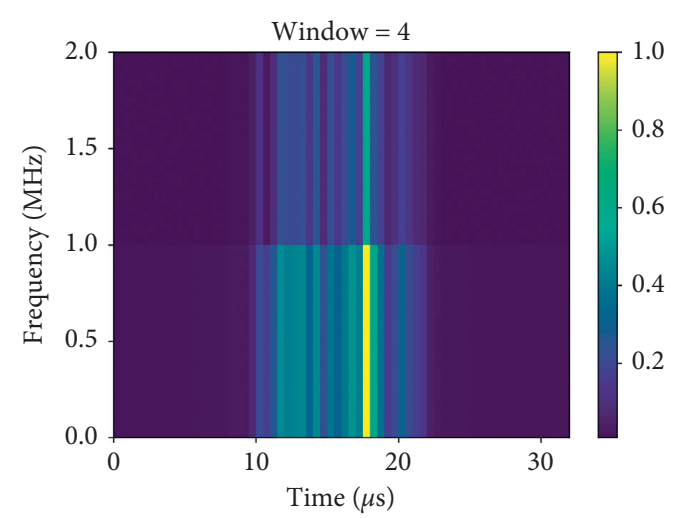

(d)

Figure 11: Time-frequency analysis diagrams under four different window lengths. (a) Time-frequency analysis diagram with a window length of 32. (b) Time-frequency analysis diagram with a window length of 16. (c) Time-frequency analysis diagram with a window length of 8. (d) Time-frequency analysis diagram with a window length of 4 .

generated by the GAN and LSGAN are very similar and model collapse has occurred.

Pearson Correlation Coefficient (PCC) and Grey Relational Analysis (GRA) are used to evaluate the quality of generated data and denoise performance of different generation models. The measured data are shown between four kinds of data and the clean HRRP, as shown in Table 2.
When the PCC is equal to 1 or the GRA is equal to 1 , the generated data are the same as the clean data, which means that the quality of the generated data and the denoise performance are great. The PCC value of the CN-LSGAN is slightly lower than the PCC value of the LSGAN, but is higher than the PCC value of the GAN. Also, the GRA value of the CN-LSGAN is much larger than that of the other two networks. From Tables 1 and 2, it can be seen that the 


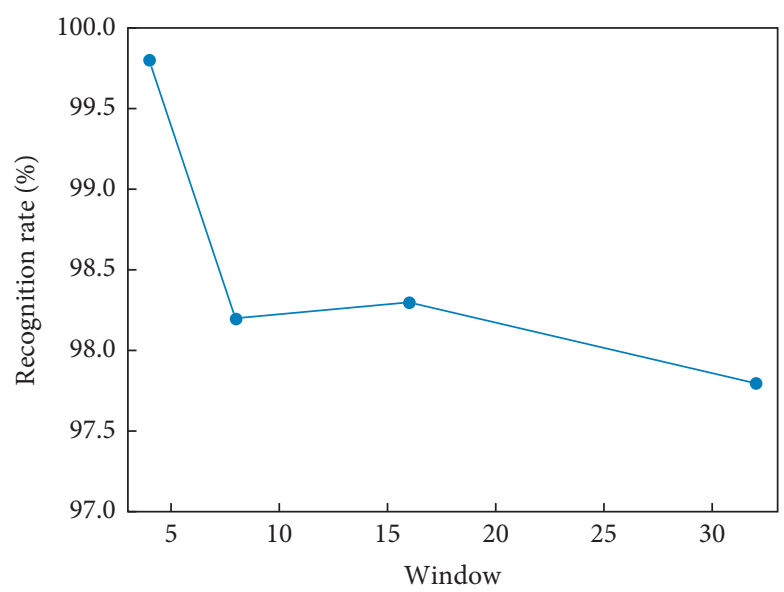

FIGURE 12: Recognition rate of time-frequency analysis results with different window lengths.

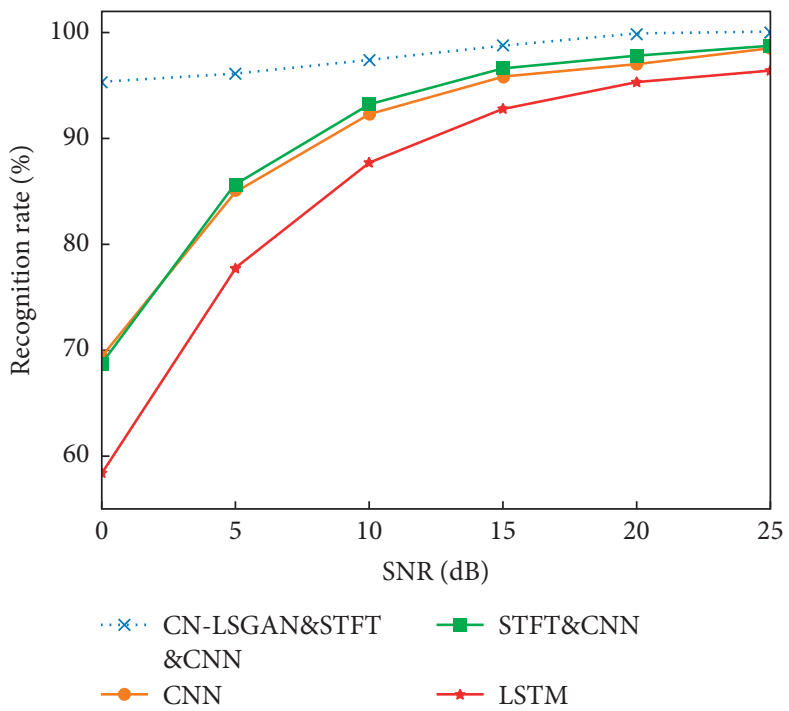

Figure 13: Recognition rate curves of four different recognition methods. The recognition rate curves showed the change trend of the recognition rate with the data signal-to-noise ratio.

CN-LSGAN not only effectively solved the model collapse problem but also generated higher data quality and better denoise performance than the GAN and LSGAN.

3.3. Time-Frequency Analysis. STFT is used for the HRRP time-frequency analysis. When the window length is large, the time resolution is poor and the frequency resolution is high. The situation is the opposite when the window length is narrow. According to the sample characteristics, the Hanning windows with window lengths of 32,16 , and 4 are used, and the obtained time-frequency data are shown in Figure 11.

Data set A performed STFT under the Hanning windows of four window lengths, and the obtained time-frequency data are recognized by the $\mathrm{CNN}$; the result is shown in Figure 12.

When the window length of the window function is 4 , the recognition rate of the obtained time-frequency analysis results can reach $99.8 \%$, so the optimal STFT window function length is 4 .

3.4. Target Recognition. The enhanced data by the CN-LSGAN are transformed into two-dimensional data by STFT and then recognized by the CNN. A variety of methods are used to recognize the noisy HRRP data (dataset $\mathrm{B}$ ), and these recognition rates are shown in Figure 13.

These recognition rates of three methods are compared to the proposed method. The first method is the one-dimensional CNN method, the second method is the LSTM method, and the third method is the STFT-CNN method, that is, STFT is combined with the two-dimensional CNN. It can be seen that the proposed method based on the CN-LSGAN, STFT, and CNN has a better recognition performance than the other three recognition methods. Also, the other three recognition methods are greatly affected by the signal-to-noise ratio, and when the 
data signal-to-noise ratio is low, these recognition rates are low. By comparing the recognition rate curve of the first method to the curve of the third method, the recognition rate of the third method is slightly lower than that of the first method when the signal-to-noise ratio is $0 \mathrm{~dB}$, while under other signal-to-noise ratios, the recognition rates are higher than the those of the first method. It shows that the third method can improve the recognition performance by combining STFT with the twodimensional CNN.

\section{Conclusions}

This paper described an HRRP target recognition method based on the CN-LSGAN, STFT, and CNN, which effectively solves the recognition problem under low signal-to-noise ratio conditions. Among them, the CN-LSGAN is obtained through adding a constraint item to $D$ and $G$ of the LSGAN, respectively. The CN-LSGAN not only solves the problems of excessive freedom degrees of GAN output, poor quality of generated data, and insignificant denoise performance but also solves the model collapse problem of the GAN and LSGAN. By the means of STFT which analyzes the time-frequency characteristics of HRRP, the frequency-domain features and phase features of the target are introduced, and more features are helpful in the feature extraction and target recognition for the CNN. These experimental results show that the method based on STFT and the $\mathrm{CNN}$ has better recognition performance compared to the method of one-dimensional CNN and the method of LSTM.

The proposed target recognition method has achieved a high recognition performance based on low signal-to-noise ratio HRRP, but there are still some problems. For example, CN-LSGAN training is unstable, and it is difficult to achieve the trade-off between the time resolution and the frequency resolution by STFT. In future research, the network structure and parameter adjustment of the CN-LSGAN will be further optimized and the Deep Convolution Generative Adversarial Network (DCGAN) will be tried to be combined with the CN-LSGAN. Also, other time-frequency analysis methods such as wavelet transform will be utilized for HRRP.

\section{Data Availability}

Experimental data are provided in supplementary materials.

\section{Conflicts of Interest}

The authors state that there are no conflicts of interest in the publication of this article.

\section{Acknowledgments}

This work was supported by the National Natural Science Foundation (61661035), Jiangxi Natural Science Foundation (20192BAB207001), and Aviation Science Foundation (201920056001).

\section{Supplementary Materials}

See Figures $1-13$ in the supplementary materials for comprehensive image analysis. For datasets $\mathrm{A}, \mathrm{B}$, and C, see text clean, $10 \mathrm{db}$, and generate in the supplementary materials. In addition, for data file description, see text document description in the supplementary materials. (Supplementary Materials)

\section{References}

[1] L. Du, P. Wang, H. Liu, M. Pan, F. Chen, and Z. Bao, "Bayesian spatiotemporal multitask learning for radar HRRP target recognition," IEEE Transactions on Signal Processing, vol. 59, no. 7, pp. 3182-3196, 2011.

[2] J. Lundén and V. Koivunen, "Deep learning for HRRP-based target recognition in multistatic radar systems," in Proceedings of the 2016 IEEE Radar Conference (RadarConf), pp. 1-6, Philadelphia, PA, USA, May 2016.

[3] K. Liao, J. Si, F. Zhu, and X. He, "Radar HRRP target recognition based on concatenated deep neural networks," IEEE Access, vol. 6, pp. 29211-29218, 2018.

[4] X. Peng, X. Gao, and X. Li, "An infinite classification RBM model for radar HRRP recognition," in Proceedings of the 2017 International Joint Conference on Neural Networks (IJCNN), pp. 1442-1448, Anchorage, Alaska, USA, May 2017.

[5] L. Shi, P. Wang, H. Liu et al., "Radar HRRP statistical recognition with local factor analysis by automatic Bayesian Ying-Yang harmony learning," IEEE Transactions on Signal Processing, vol. 59, no. 2, pp. 610-617, 2010.

[6] S. Luo and S. Li, "Automatic target recognition of radar HRRP based on high order central moments features," Journal of Electronics (China), vol. 26, no. 2, pp. 184-190, 2009.

[7] B. Feng, B. Chen, and H. Liu, "Radar HRRP target recognition with deep networks," Pattern Recognition, vol. 61, pp. 379-393, 2017.

[8] D. Zhou, "Radar target HRRP recognition based on reconstructive and discriminative dictionary learning," Signal Processing, vol. 126, pp. 52-64, 2016.

[9] C. Guo, Y. He, H. Wang, T. Jian, and S. Sun, "Radar HRRP target recognition based on deep one-dimensional residual-inception network," IEEE Access, vol. 7, pp. 9191-9204, 2019.

[10] H. Zhang, D. Ding, Z. Fan et al., "Adaptive neighborhood-preserving discriminant projection method for HRRP-based radar target recognition," IEEE Antennas and Wireless Propagation Letters, vol. 14, pp. 650-653, 2014.

[11] L. Du, H. Liu, Z. Bao et al., "A two-distribution compounded statistical model for radar HRRP target recognition," IEEE Transactions on Signal Processing, vol. 54, no. 6, pp. 2226-2238, 2006.

[12] M. Pan, J. Jiang, Q. Kong, J. Shi, Q. Sheng, and T. Zhou, "Radar HRRP target recognition based on t-SNE segmentation and discriminant deep Belief network," IEEE Geoscience and Remote Sensing Letters, vol. 14, no. 9, pp. 1609-1613, 2017.

[13] J. Li, S. Li, Q. Liu et al., "A novel algorithm for HRRP target recognition based on CNN," in Proceedings of the International Conference on Internet of Things as a Service, pp. 397-404, Springer, Diego, CA, USA, June 2019.

[14] J. Song, Y. Wang, W. Chen, Y. Li, and J. Wang, "Radar HRRP recognition based on CNN," The Journal of Engineering, vol. 2019, no. 21, 7769 pages, 2019.

[15] B. Xu, B. Chen, J. Wan, H. Liu, and L. Jin, "Target-aware recurrent attentional network for radar HRRP target recognition," Signal Processing, vol. 155, pp. 268-280, 2019.

[16] V. Jithesh, M. J. Sagayaraj, and K. G. Srinivasa, "LSTM recurrent neural networks for high resolution range profile based radar target classification," in Proceedings of the 2017 $3 r d$ International Conference on Computational Intelligence \& 
Communication Technology (CICT), pp. 1-6, IEEE, Athens, Greece, July 2017.

[17] D. Li, S. Gong, S. Niu, Z. Wang, D. Zhou, and H. Lu, "Image blind denoising using a generative adversarial network for LED chip visual localization," IEEE Sensors Journal, vol. 20, no. 12, pp. 6582-6595, 2020.

[18] T. Kaneko, H. Kameoka, N. Hojo et al., "Generative adversarial network-based postfilter for statistical parametric speech synthesis," in Proceedings of the 2017 IEEE International Conference on Acoustics, Speech and Signal Processing (ICASSP), pp. 4910-4914, IEEE, Orleans, LA, USA, March 2017.

[19] S. Pascual, A. Bonafonte, and J. Serrà, "SEGAN: speech enhancement generative adversarial network," in Proceedings of the Annual Conference of the International, Austin, Texas, Janurary 2017.

[20] C. Zhao, X. He, J. Liang, T. Wang, and C. Huang, "Radar HRRP target recognition via semi-supervised multi-task deep network," IEEE Access, vol. 7, pp. 114788-114794, 2019.

[21] I. Goodfellow, J. Pouget-Abadie, M. Mirza et al., "Generative adversarial network," Communications of the ACM, vol. 63, no. 11, pp. 139-144, 2014.

[22] X. Mao, Q. Li, H. Xie et al., "Least squares generative adversarial networks," in Proceedings of the IEEE International Conference on Computer Vision, pp. 2794-2802, Venice, Italy, October 2017. 